\title{
Aspectos ecológicos da fauna de flebotomíneos em focos de leishmaniose na Amazônia Oriental, Estado do Pará, Brasil
}

\section{Ecological aspects of phlebotomine sand flies in foci of leishmaniasis in the eastern Amazon, Pará State, Brazil}

Anadeiva Portela Chagas', Daniela Cristina Soares², Gilberto Cesar Rodrigues de Sousa ${ }^{1}$, Rosangela Barros Viana ${ }^{1}$, José Manuel Macário Rebelo3, Lourdes Maria Garcez ${ }^{1,4}$

\author{
1 Instituto Evandro Chagas/SVS/MS, Seção de Parasitologia, Ananindeua, Pará, Brasil \\ 2 Secretaria de Vigilância em Saúde/MS, Brasília, Distrito Federal, Brasil \\ ${ }^{3}$ Universidade Federal do Maranhão, São Luís, Maranhão, Brasil \\ ${ }^{4}$ Universidade do Estado do Pará, Belém, Pará, Brasil
}

\section{RESUMO}

Este estudo descreve comunidades de flebotomíneos e suas variações sazonais em focos de transmissão das leishmanioses na interface urbano-rural do município minerário de Juruti, Estado do Pará, na Região Amazônica. Realizou-se captura mensal de flebotomíneos durante dois anos, em duas localidades sentinelas, Santa Maria e Paraense. Armadilhas CDC foram colocadas no intra e no peridomicílio. A amostra de 36.408 flebotomíneos teve 32 espécies representadas; as mais frequentes foram Lutzomyia longipalpis (76,8\%) e Lutzomyia walkeri (19\%). A riqueza de espécies foi maior em Paraense, a despeito da destacada abundância de Lu. longipalpis (85\%, 23.878/27.951), sobretudo no inverno amazônico $(r=0,8 ; p<0,05)$. Em Santa Maria, Lu. longipalpis, pouco frequente, não apresentou variação sazonal. Constatou-se a ocorrência, apenas em Paraense, no interior de residências, de flebotomíneos naturalmente infectados por Leishmania sp.: Lu. longipalpis (0,12\%; 1/821), vetor de Leishmania (Leishmania) infantum; e Lutzomyia antunesi (1,16\%; 2/173), provável vetor de Leishmania (Viannia) lindenbergi. A alta frequência de Lu. longipalpis no período chuvoso, mas apenas em Paraense, revela influências locais determinantes da composição da comunidade de flebotomíneos e abundância de espécies. Ações integradas para a prevenção das leishmanioses devem ser contínuas e preferencialmente intensificadas de setembro a novembro, meses que antecedem as chuvas naquela região.

Palavras-chave: Leishmaniose; Região Amazônica; Psychodidae; Insetos Vetores; Ecologia.

\begin{abstract}
This study describes the phlebotomine communities and their seasonal variation in areas of leishmaniasis transmission in the urban-rural interface of the mining municipality of Juruti, Pará State, in the Amazon Region. Monthly captures of phlebotomine sand flies were performed over two years in two sentinel locations: Santa Maria and Paraense. CDC traps were placed on intra and peridomestic environments. A sample of 36,408 phlebotomine sand flies contained 32 species. The most frequent were Lutzomyia longipalpis (76.8\%) and Lutzomyia walkeri (19\%). Species richness was greater in Paraense, despite the abundant presence of Lu. longipalpis (85\%, 23,878/27,951), especially during the Amazonian winter $(r=0.8 ; p<0.05)$. In Santa Maria, the presence of Lu. longipalpis, which occurs rarely, showed no seasonal variation. In the interior of residences, exclusively in Paraense, there was also the occurrence of phlebotomine sand flies naturally infected by Leishmania sp.: Lu. longipalpis $(0.12 \%$; 1/821), vector of Leishmania (Leishmania) infantum and Lutzomyia antunesi $(1.16 \% ; 2 / 173)$, most likely the vector of Leishmania (Viannia) lindenbergi. The high frequency of Lu. longipalpis during the rainy season, but only in Paraense, reveals local influences that determine the phlebotomine community composition and the abundance of species. Integrated actions for the prevention of leishmaniasis must be continuous and preferably intensified from September to November, months preceding rainfall in that region.
\end{abstract}

Keywords: Leishmaniasis; Amazon Region; Psychodidae; Vector Insects; Ecology.

\footnotetext{
Correspondência / Correspondence:

Anadeiva Portela Chagas

Instituto Evandro Chagas/SVS/MS, Seção de Parasitologia, Laboratório de Epidemiologia e Imunologia das Leishmanioses Rodovia BR-316 km 7, s/n. Bairro: Levilândia - CEP: 67030-000 - Ananindeua, Pará, Brasil - Tel.: +55 (91) $3214-2152$

E-mail: anadeivaportela@hotmail.com
} 


\section{INTRODUÇÃO}

Os flebotomíneos apresentam uma elevada riqueza de espécies na Região Amazônica', com altos índices de diversidade local em florestas de terra firme ${ }^{2}$. Das mais de 230 espécies de Lutzomyia já identificadas no Brasil, 122 podem ser encontradas na Amazônia. Mais de 20 espécies picam o ser humano e, consequentemente, várias delas têm importância como vetores de leishmaniose $e^{3,4,5}$

A composição da fauna de flebotomíneos do gênero Lutzomyia é bem estudada no Estado do Pará. Até o presente momento, tem-se registro da existência de pelo menos 117 espécies $^{6}$ para as quais existe chave regional de identificação ${ }^{7}$. Esse conhecimento deriva principalmente de estudos do papel desses insetos como vetores de Leishmania, o que contribuiu para desvendar alguns aspectos da ecoepidemiologia das leishmanioses na Amazônia brasileira $8,9,10,11$. Entretanto, os estudos de levantamentos faunísticos sistematizados e duradouros ainda são incipientes. Nas áreas silvestres, tem-se conhecimento de trabalhos que estudaram a fauna de mata de terra firme e várzea das margens do rio Capim, Município de Paragominas ${ }^{12}$; inquéritos entomológicos na Serra do Carajás, Mesorregião do Sudeste Paraense ${ }^{13}$; e na Floresta Nacional do Tapajós, Município de Belterra, no Baixo Amazonas ${ }^{14}$. Em áreas antropizadas, há alguns estudos sobre a riqueza, abundância e distribuição horizontal da fauna em áreas urbanas e rurais ${ }^{15,16}$. Outros levantamentos foram realizados em fragmentos florestais da área metropolitana de Belém ${ }^{17}$. Esses estudos confirmam a grande diversidade e abundância de flebotomíneos presentes na Região Amazônica.

A existência de uma rica fauna de flebotomíneos no Pará justifica a situação endêmica das leishmanioses nesse território. Sabe-se que tanto a leishmaniose tegumentar (LT) como a leishmaniose visceral (LV) vêm adquirindo importância em números de casos e de municípios afetados. Essa situação decorre, entre outros fatores, da rápida ocupação dos territórios por diversas razões, incluindo a implantação de projetos agrícolas e minerários ${ }^{18}$, pois a composição e a riqueza das populações de vetores são resultados de interações ecológicas e fatores geográficos em uma área ${ }^{19}$. Assim, mudanças no ambiente afetam a dinâmica das populações de flebotomíneos, alteram sua composição, seus hábitos e o comportamento das diferentes espécies, bem como a sua capacidade vetorial $^{20}$.

No oeste do Pará, a riqueza mineral da região do Tapajós atrai, da parte de investidores, empresas nacionais e estrangeiras, grande atenção para essa área amazônica. Nessa região, destaca-se o Município de Juriti, que possui uma das maiores reservas de bauxita do mundo, com projeção de extração para os próximos $45 \operatorname{anos}^{21}$. A construção de um empreendimento pela mineradora Alcoa S/A se deu em 2006, quando também se iniciaram estudos sobre as leishmanioses ${ }^{22,23}$ como incentivos do seu plano de controle ambiental, em virtude do aumento do número de casos de LT registrados na época. Como exemplo, cita-se a taxa de incidência, que era de 38,6 casos/100.000 habitantes em 2003, elevando para 65, em 2004, e para 138 em 2005. Inquéritos entomoepidemiológicos, realizados por Garcez et $a^{23}$ em Juruti, revelaram a presença de reservatórios domésticos infectados e alta abundância de Lutzomyia longipalpis no peridomicílio de áreas periurbanas desse Município. Neste trabalho, estudou-se a fauna de flebotomíneos de duas localidades periurbanas de Juruti, com os objetivos de se determinar a abundância e a diversidade de espécies e de se discutirem aspectos ecológicos relacionados à transmissão da leishmaniose nessa área.

\section{MATERIAIS E MÉTODOS}

\section{ÁREA DE ESTUDO}

O Município de Juruti situa-se no Estado do Pará, na divisa com o Estado do Amazonas. Possui uma população estimada de 53.989 habitantes e 8.304 km² de área ${ }^{24}$.

Predominam na região florestas densas dos baixos platôs e campos cerrados. As temperaturas anuais variam de $22,5^{\circ} \mathrm{C}$ a $31^{\circ} \mathrm{C}$, com médias de $25,6^{\circ} \mathrm{C}$. As chuvas são irregulares. Contudo, os meses de dezembro a maio correspondem à estação chuvosa, e de junho a novembro, à estação seca, conhecidas, respectivamente, como inverno e verão amazônico. A precipitação anual acumulada chega a $2.000 \mathrm{~mm}$ e a umidade relativa apresenta valores acima de $80 \%$ em quase todos os meses do $a_{n o}{ }^{24}$. Para as capturas entomológicas, foram selecionadas duas localidades situadas em uma área indiretamente impactada pelo empreendimento supracitado, na interface urbano-rural, distante $12 \mathrm{~km}$ da sede do Município: Santa Maria $\left(2^{\circ} 11^{\prime} 17,5^{\prime \prime} S\right.$; 560.'42,7"W) e Paraense $\left(2^{\circ} 09^{\prime} 14,7^{\prime \prime} S\right.$; $\left.56^{\circ} 00^{\prime} 29,6^{\prime \prime} \mathrm{W}\right)$.

$\mathrm{Na}$ localidade de Santa Maria, com aproximadamente 303 habitantes, predominavam utensílios e organização de cultura indígena e quilombola, com espaços mais abertos e pouca vegetação entre as casas, matas e água. No entorno da comunidade, a ocupação do solo era destinada principalmente para a agricultura de subsistência. Já na localidade de Paraense, com apenas 94 habitantes, predominava vegetação do tipo secundária, com casas construídas em áreas de capoeiras abertas. Os terrenos eram sombreados por uma grande quantidade de árvores frutíferas e os abrigos de animais construídos nas proximidades das residências $(<10 \mathrm{~m})$. Nas casas, à beira do rio, havia a presença de vãos entre o assoalho e o terreno, que podiam ser utilizados como abrigos pelos animais domésticos e insetos vetores. Nessa localidade, os moradores disseram ter visto reservatórios silvestres. 

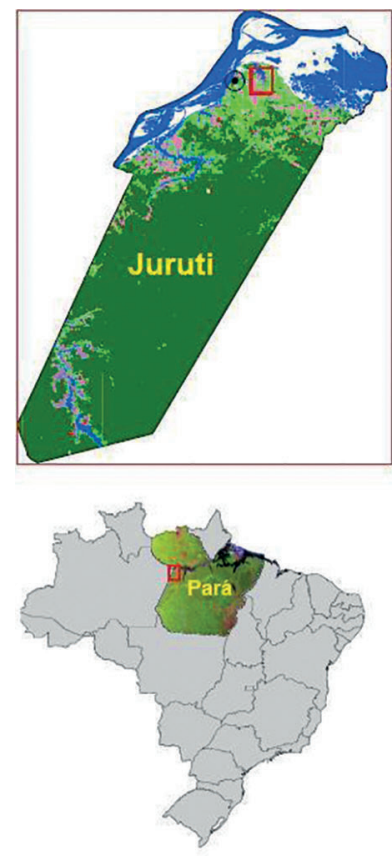

LEGENDA

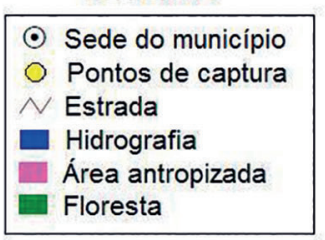

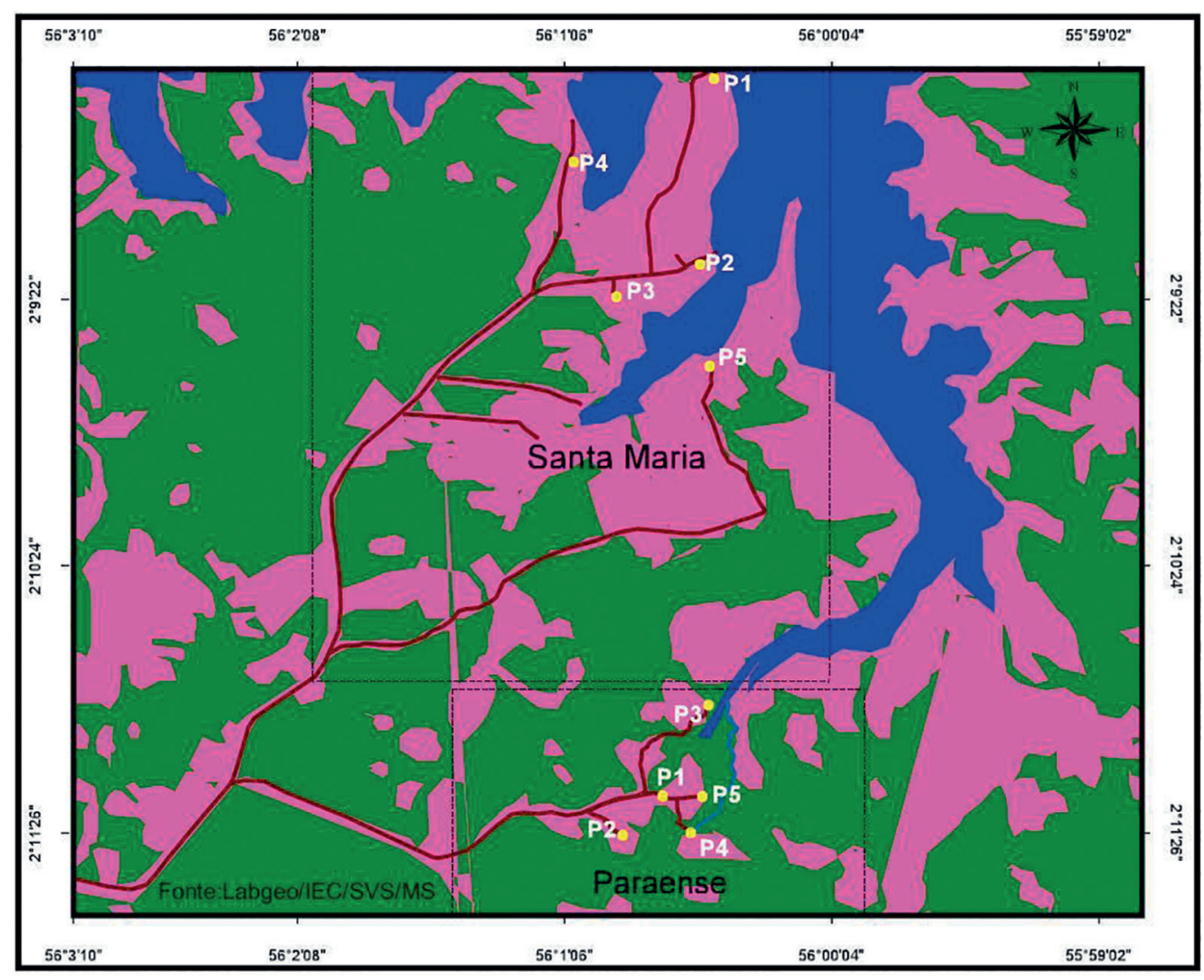

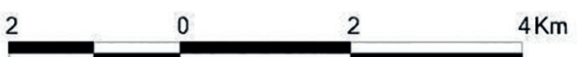

Fonte: Laboratório de Geoprocessamento/IEC/SVS/MS

Figura 1 - Mapa do Município de Juruti, Estado do Pará, Brasil, destacando os pontos de capturas entomológicas (P) nas localidades de Santa Maria e Paraense

\section{CAPTURAS ENTOMOLÓGICAS E DETERMINAÇÃO DA INFECÇÃO NATURAL}

Em cada localidade, utilizando critérios recomendados pelo Ministério da Saúde ${ }^{25}$, cinco pontos (residências) foram selecionados para a realização das capturas mensais de flebotomíneos (julho/2009 a junho/2011) (Figura 1). Nessas residências, foram instaladas duas armadilhas luminosas tipo CDC: uma no intradomicílio e outra no peridomicílio, a uma altura de $1,5 \mathrm{~m}$ do chão. As armadilhas foram instaladas das 18 às $6 \mathrm{~h}$, durante três noites consecutivas. Os insetos capturados foram separados em frascos de vidro e encaminhados ao laboratório. Todos os espécimes foram conservados em álcool etílico (70\%), para posterior montagem em lâmina com solução de Berlese e identificação, de acordo com a chave de identificação de Young e Duncan'. Para determinação da taxa de infecção natural, foram dissecadas fêmeas de flebotomíneos a fresco em solução. O intestino foi examinado ao microscópio óptico, em aumento de 400 x, para visualização de possíveis parasitos $^{26}$.

\section{ANÁLISE ESTATÍSTICA}

Os perfis de diversidade pela série de Rényi, que fornece diferentes medidas de diversidade para comparar, de forma equivalente, os locais de captura, foram calculados utilizando o pacote Vegan do programa estatístico R.2.1027, sendo o valor de diversidade igual ao número de espécies da amostra quando o parâmetro $\alpha=0$. Para $\alpha=1$, o valor da diversidade corresponde ao índice de Shannon; e, se considerado $\alpha=2$, ao índice de Simpson ${ }^{28}$. A análise de ordenação NMDS (Escalonamento Multidimensional Não Métrico), usando-se o índice de dissimilaridade Bray-Curtis, foi utilizada para comparar a composição da fauna de flebotomíneos entre os pontos de captura. No programa BioEstat v5.029, foi realizado o teste Kruskal-Wallis para avaliar diferenças na abundância de indivíduos entre os diferentes locais de captura, e determinada a correlação entre a abundância de flebotomíneos capturados e os valores mensais da precipitação pluviométrica por meio da correlação linear de Pearson $(\alpha=0,01)$.

\section{RESULTADOS}

\section{RIQUEZA E DIVERSIDADE DE ESPÉCIES}

Considerando as duas localidades, foi capturado um total de 32 espécies, sendo a riqueza maior em Paraense (31) do que em Santa Maria (15) (Tabela 1). O perfil Rényi revelou que, embora a riqueza $(\alpha=0)$ tenha sido maior no peri e no intradomicílio de Paraense, respectivamente, os índices de Shannon $(\alpha=1)$ e Simpson $(\alpha=2)$ revelaram que a maior diversidade foi encontrada no peridomicílio de Santa Maria (Figura 2). 
Tabela 1 - Número de espécimes de flebotomíneos capturados nos ambientes intra e peridomiciliar das localidades de Santa Maria e Paraense, no Município de Juriti, Estado do Pará, Brasil, de julho/2009 a junho/201 1

\begin{tabular}{|c|c|c|c|c|c|c|c|c|}
\hline \multirow{2}{*}{ Espécies } & \multicolumn{3}{|c|}{ Santa Maria } & \multicolumn{3}{|c|}{ Paraense } & \multicolumn{2}{|c|}{ Total } \\
\hline & Peridomicílio & Intradomicílio & Subtotal & Peridomicílio & Intradomicílio & Subtotal & № & $\%$ \\
\hline Lu. longipalpis & 3.609 & 464 & 4.073 & 20.469 & 3.409 & 23.878 & 27.951 & 76,77 \\
\hline Lu. walkeri & 2.981 & 3.924 & 6.905 & 331 & 19 & 350 & 7.255 & 19,93 \\
\hline Lu. antunesi & 14 & 2 & 16 & 423 & 131 & 554 & 570 & 1,57 \\
\hline Lu. williamsi & 3 & 1 & 4 & 113 & 17 & 130 & 134 & 0,37 \\
\hline Lu. sordellii & 5 & 3 & 8 & 60 & 35 & 95 & 103 & 0,28 \\
\hline Lu. pinottii & - & - & - & 68 & 23 & 91 & 91 & 0,25 \\
\hline Lu. saulensis & 18 & 4 & 22 & 51 & 9 & 60 & 82 & 0,23 \\
\hline Lu. flaviscutellata & 4 & 1 & 5 & 27 & 11 & 38 & 43 & 0,12 \\
\hline Lu. shannoni & 5 & - & 5 & 24 & 2 & 26 & 31 & 0,09 \\
\hline Lu. ubiquitalis & 2 & - & 2 & 19 & 9 & 28 & 30 & 0,08 \\
\hline Lu. abonnenci & 4 & 2 & 6 & 12 & - & 12 & 18 & 0,05 \\
\hline Lu. furcata & - & - & - & 10 & 7 & 17 & 17 & 0,05 \\
\hline Lu. lutziana & 5 & 4 & 9 & 4 & 1 & 5 & 14 & 0,04 \\
\hline Lu. wilsoni & - & - & - & 9 & - & 9 & 9 & 0,02 \\
\hline Lu. davisi & - & - & - & 8 & 1 & 9 & 9 & 0,02 \\
\hline Lu. ayrozai & - & - & - & 6 & 2 & 8 & 8 & 0,02 \\
\hline Lu. bacula & - & - & - & 5 & 1 & 6 & 6 & 0,02 \\
\hline Lu. longispina & - & - & - & 5 & 1 & 6 & 6 & 0,02 \\
\hline Lu. evandroi & 3 & - & 3 & 2 & - & 2 & 5 & 0,01 \\
\hline Lu. shawi & - & - & - & 5 & - & 5 & 5 & 0,01 \\
\hline Lu. rorotaensis & - & - & - & 2 & 1 & 3 & 3 & 0,01 \\
\hline Lu. carrerai & - & - & - & 2 & - & 2 & 2 & 0,01 \\
\hline Lu. chagasi & 1 & - & 1 & - & 1 & 1 & 2 & 0,01 \\
\hline Lu. wellcomei & - & - & - & 1 & 1 & 2 & 2 & 0,01 \\
\hline Lu. monstruosa & - & - & - & 2 & - & 2 & 2 & 0,01 \\
\hline Lu. carmelinoi & - & 1 & 1 & 1 & - & 1 & 2 & 0,01 \\
\hline Lu. paraensis & - & - & - & 2 & - & 2 & 2 & 0,01 \\
\hline Lu. richardwardi & - & - & - & 2 & - & 2 & 2 & 0,01 \\
\hline Lu. dasymera & - & - & - & 1 & - & 1 & 1 & 0,00 \\
\hline Lu. inpai & - & - & - & 1 & - & 1 & 1 & 0,00 \\
\hline Lu. elegans & - & - & - & - & 1 & 1 & 1 & 0,00 \\
\hline Lu. servulolimai & 1 & - & 1 & - & - & - & 1 & 0,00 \\
\hline Total de indivíduos & 6.655 & 4.406 & 11.061 & 21.665 & 3.682 & 25.347 & 36.408 & 100,00 \\
\hline Total de espécies & 14 & 10 & 15 & 29 & 20 & 31 & 32 & \\
\hline
\end{tabular}

Sinal convencional utilizado: - Dado numérico igual a zero não resultante de arredondamento. 


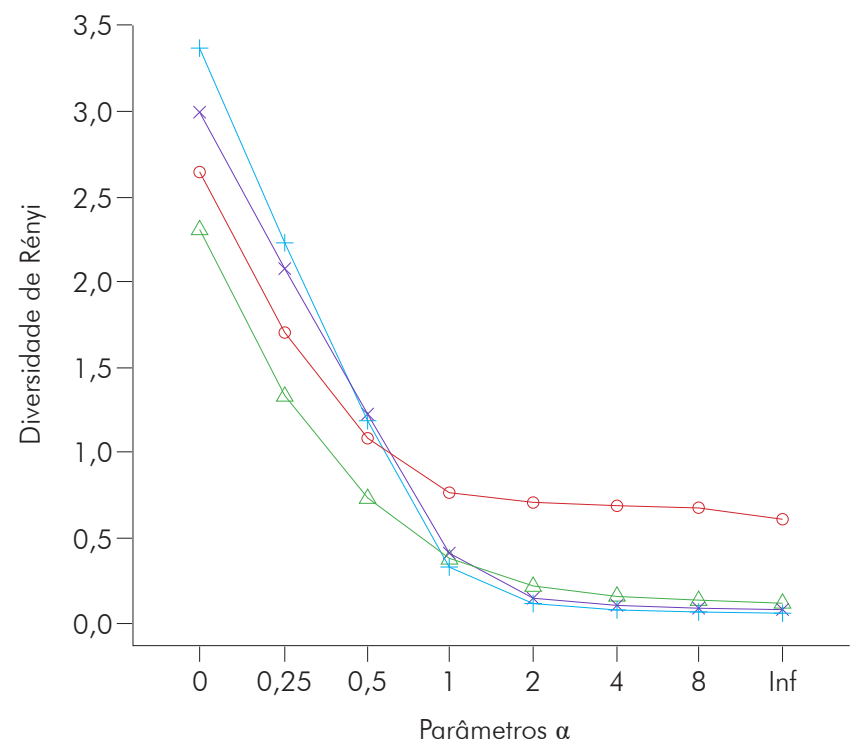

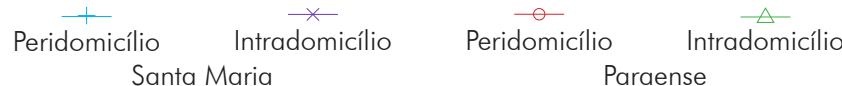
Santa Maria

Figura 2 - Riqueza de espécies $(\alpha=0)$ e perfis de diversidade de Rényi, mostrando os índices de diversidade de Shannon $(\alpha=1)$ e Simpson $(\alpha=2)$, nos peridomicílios e intradomicílios das localidades de Santa Maria e Paraense, no Município de Juriti, Estado do Pará, Brasil (julho/2009 a junho/2011)

\section{ABUNDÂNCIA RELATIVA}

estudo resultou na captura de um total de 36.408 flebotomíneos nas duas localidades. A espécie mais abundante foi Lu. longipalpis, com 27.951 espécimes $(76,7 \%)$, seguida por Lutzomyia walkeri (7.256; 19,9\%). Essas duas espécies somaram 96,6\% do total de flebotomíneos capturados durante o período amostral. $\bigcirc$ restante (3,4\%) ficou distribuído entre 30 espécies. Em Santa Maria, foram capturados 11.061 flebotomíneos pertencentes a 15 espécies. A mais abundante foi Lu. walkeri (6.906; 62,4\%), seguida de Lu. longipalpis (4.073; 36,8\%) (Tabela 1). Em Paraense, foram capturados 25.347 flebotomíneos de 31 espécies, sendo Lu. longipalpis a mais abundante (23.878; 85\%), seguida de Lutzomyia antunesi (554; 2,2\%) (Tabela 1). Além da alta abundância de Lu. longipalpis, em Paraense, foi observada a presença de seis das oito espécies de flebotomíneos envolvidas na transmissão de LT na Amazônia5: Lu. antunesi, Lutzomyia flaviscutellata, Lutzomyia ubiquitalis, Lutzomyia wellcomei, Lutzomyia ayrozai e Lutzomyia paraensis. Já em Santa Maria, em menor frequência, foram identificadas apenas as espécies Lu. antunesi, Lu. flaviscutellata e Lu. ubiquitalis (Tabela 1). Conforme a figura 3, utilizando-se a ordenação dos pontos amostrais por NMDS, verificou-se que houve diferença entre as duas localidades tanto na composição das espécies $(F=4,91 ; p<0,001)$ como na abundância relativa das populações $(F=3,3 ; p<0,05)$. $\bigcirc$ teste Kruskal-Wallis detectou diferenças significativas na abundância dos flebotomíneos entre os ambientes intra e peridomiciliar $(H=37,9 ; p<0,001)$ e os pontos de captura $(H=18,801 ; p<0,001)$, sendo maior no peridomicílio do que no intradomicílio, tanto em Paraense $(85,5 \%$; 14,5\%) quanto em Santa Maria $(60,2 \% ; 39,8 \%)$. Neste estudo, o número de machos superou o de fêmeas em todo o período amostral, mas essa variação não foi considerada estatisticamente significante.

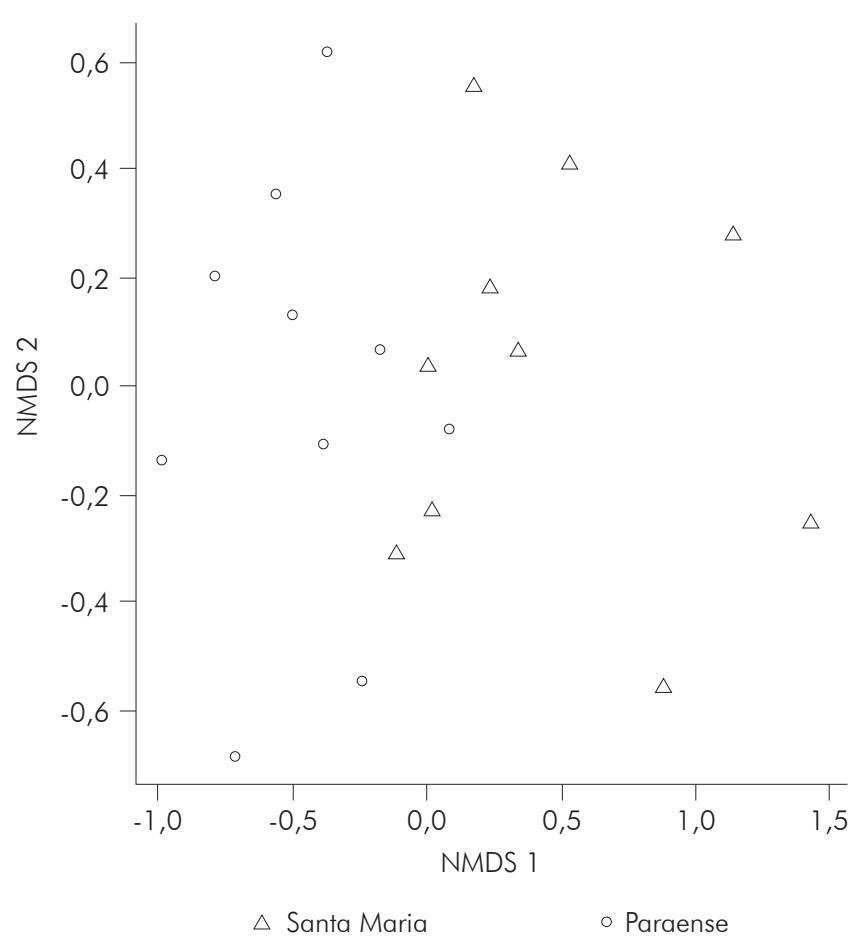

Figura 3 - Ordenação dos pontos amostrais por NMDS da ocorrência das espécies nas localidades de Santa Maria e Paraense, no Município de Juriti, Estado do Pará, Brasil (julho/2009 a junho/2011)

\section{DISTRIBUIÇÃO SAZONAL}

Os flebotomíneos predominaram na estação chuvosa nas duas localidades (Figura 4). Em Paraense, 15 espécies ocorreram nas duas estações; 13 só foram encontradas na estação chuvosa; e três apenas na seca. Em Santa Maria, oito espécies ocorreram nas duas estações; quatro somente na chuvosa; e três apenas na seca. $\bigcirc$ teste de Kruskal-Wallis detectou diferenças significativas na abundância dos flebotomíneos entre as estações $(H=26,85 ; \mathrm{p}<$ $0,001)$ para a localidade de Paraense, na qual a abundância de Lu. longipalpis esteve correlacionada positivamente aos níveis de precipitação pluviométrica mensais $(r=0,82 ; p<0,01)$ (Figura 5).

\section{INFECÇÃO NATURAL}

Durante os 24 meses de capturas, foram dissecadas 1.933 fêmeas de flebotomíneos 1.348 de Paraense e 585 de Santa Maria. Foram encontradas três fêmeas infectadas com Leishmania sp. no intradomicílio de Paraense, sendo um exemplar de Lu. longipalpis e dois de Lu. antunesi, o que representou uma taxa de infecção de 0,12\% e 1,16\%, respectivamente. 


\section{A}



B

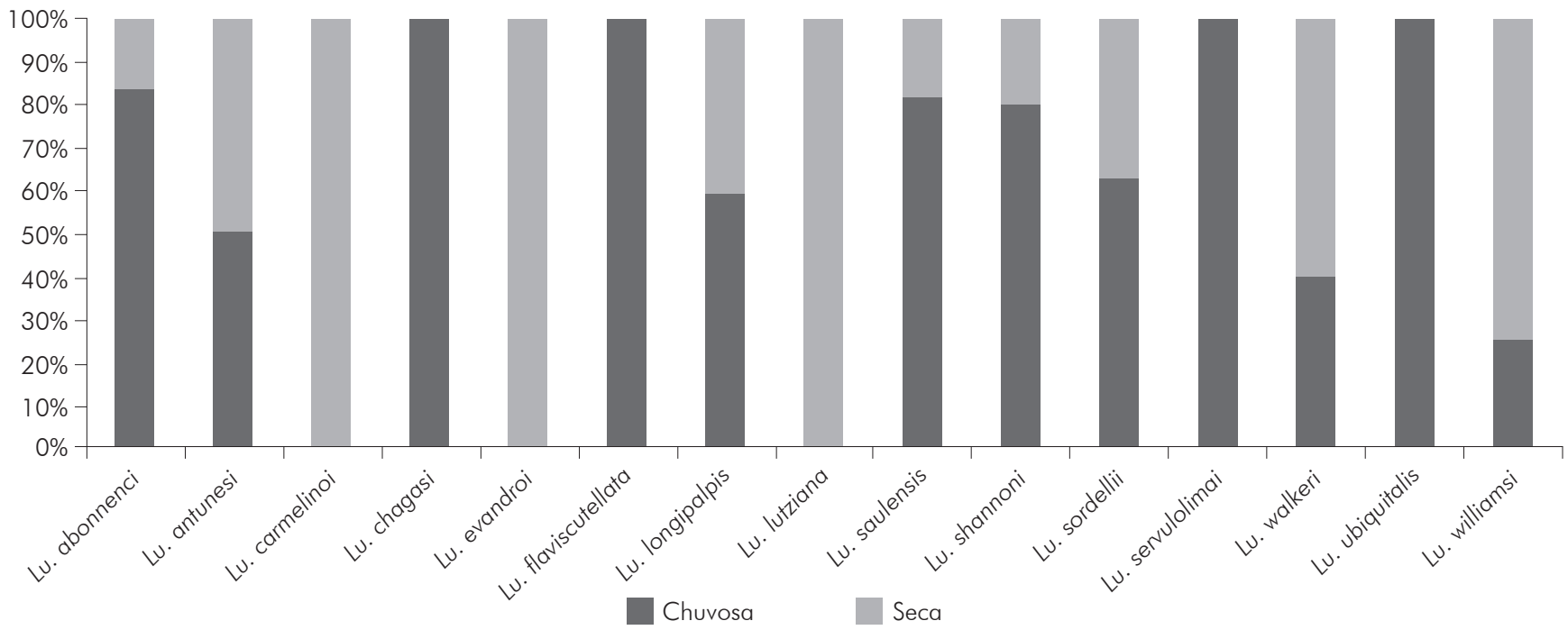

Figura 4 - Números percentuais de espécimes de flebotomíneos capturados nas estações chuvosa e seca, nas localidades de Paraense (A) e Santa Maria (B), no Município de Juriti, Estado do Pará, Brasil (julho/2009 a junho/201 1)

\section{DISCUSSÃO}

Neste estudo, a riqueza de espécies foi considerada elevada, confirmando observações anteriores em áreas florestais da Amazônia paraense ${ }^{12,13,15,16}$. Apesar da grande riqueza, a maioria das espécies contribuiu com um número baixíssimo de indivíduos. Apenas duas foram consideradas dominantes: Lu. longipalpis, no peridomicílio de Paraense; e Lu. walkeri, no intradomicílio de Santa Maria. Resultados semelhantes foram descritos em ambiente florestal da Amazônia maranhense ${ }^{30}$. Essa baixa frequência de indivíduos estaria relacionada à interação de um conjunto de fatores, mas principalmente à ausência de fontes alimentares sanguíneas específicas para os flebotomíneos e à presença dos seus criadouros e abrigos no raio de atração das armadilhas ${ }^{31}$. Por outro lado, algumas espécies poderiam estar em fase inicial de adaptação ao ambiente peridoméstico. Tais espécies, ainda que aparentemente raras (Tabela 1) e sem importância conhecida na epidemiologia das leishmanioses, seriam passíveis de adaptação e de virem a desempenhar funções vetoriais ${ }^{32}$.
Entre as localidades, a riqueza e abundância das espécies foram diferenciadas. Isso sugere que fatores ambientais ou geográficos devem estar determinando a estruturação diferente das comunidades. Assim, as maiores riqueza e abundância de flebotomíneos em Paraense podem ser justificadas pelo fato de essa localidade manter suas características rurais, com presença de vasta vegetação secundária, por exemplo; ao contrário de Santa Maria, que é mais urbanizada e apresenta áreas com solo exposto utilizado para a agropecuária. Além disso, deve-se considerar também o estilo das habitações humanas e das atitudes e práticas dessa comunidade. As casas construídas em áreas de capoeiras abertas para abrigar residências, os terrenos sombreados por uma grande quantidade de árvores frutíferas e os abrigos de animais construídos perto das residências (< $10 \mathrm{~m}$ ), bem como a ausência de segregação dos espaços de trabalho dentro das casas, são fatores importantes, que podem contribuir para a manutenção de criadouros e fonte de alimentos para os vetores. 



Figura 5 - Número de flebotomíneos capturados nas localidades de Paraense (A) e Santa Maria (B), de julho de 2009 a junho de 2011 , e os índices de precipitação pluvial para o mesmo período no Município de Juruti, Estado do Pará, Brasil

A dominância de Lu. longipalpis em relação às demais espécies foi demonstrada em diversos estudos já realizados no Pará ${ }^{33}$, Amazonas ${ }^{34}$, Minas Gerais $^{35}$, Rio Grande do Norte ${ }^{36}$ e Mato Grosso ${ }^{37,38}$. O alto grau de adaptação de Lu. longipalpis ao ambiente peridoméstico de áreas antropizadas é influenciado, principalmente, pela presença de animais domésticos ${ }^{36,39,40}$. Alexander et al ${ }^{41}$ demonstraram 0 importante papel que galinhas possuem para atrair Lu. longipalpis para a proximidade do peridomićlio. No entanto, em Paraense, além da criação de galinhas, observada em todos os pontos monitorados, em um ponto, que capturou $56 \%$ do total de flebotomíneos dessa localidade (14.424/25.355), havia, também, um chiqueiro de porcos. Santini et al ${ }^{42}$ relataram que a existência de abrigos de animais no peridomićlio aumenta três vezes a chance de haver maior abundância vetorial nesse ambiente.

Em Paraense, apenas 14,5\% das espécies foram capturadas no intradomićlío. Em Santa Maria, esse número foi elevado a $39,4 \%$, principalmente devido à grande quantidade de machos de Lu. walkeri nesse ambiente. Lu. walkeri já foi descrita em áreas, de floresta na Amazônia ${ }^{43,44,45}$ e no Nordeste do pais ${ }^{46}$, mas nenhuma importância epidemiológica the foi atribuída.

Os inquéritos entomológicos levados a efeito no

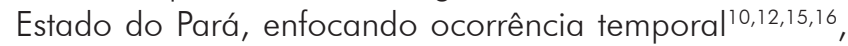
revelaram que os flebotomíneos podem ser encontrados em qualquer época do ano. Entretanto, a maior abundância de indivíduos no período chuvoso corrobora os estudos realizados em várias regiões do Brasi $^{47,48,49,50}$. De acordo com Rutledge e Ellenwood ${ }^{51}$, as chuvas agem modificando e prejudicando as condições dos criadouros no solo, mas aumentam a umidade do ar, favorecendo as atividades dos adultos.

O estudo da infecção por Leishmania sp. revelou positividade em Lu. longipalpis e Lu. antunesi, duas espécies de flebotomíneos relacionadas com a transmissão de LV e LT, respectivamente. Esse resultado sugere que a leishmaniose estava sendo transmitida dentro da própria localidade. A julgar pelo ambiente onde os exemplares foram encontrados infectados, a 
transmissão pode ocorrer, inclusive, dentro de casa. Convém mencionar que, em Paraense, além de Lu. longipalpis, vetor da LV, foi observada a presença de seis das oito espécies de flebotomíneos implicadas na transmissão de LT na Amazônia ${ }^{5}$.

\section{CONCLUSÃO}

A maior abundância e riqueza de espécies de flebotomíneos foram encontradas em Paraense. Nessa localidade, Lu. longipalpis foi a espécie mais abundante e dominante e sua distribuição sazonal foi correlacionada ao aumento dos níveis de precipitação pluvial. A abundância e a presença de vetores naturalmente infectados chamam atenção para a possibilidade de aumento do número de casos de LV e LT, não apenas nessa localidade, mas também em outras áreas rurais expostas ao impacto direto de transformações ambientais resultantes de atividades minerárias. Os resultados orientam ações de vigilância, prevenção e controle das leishmanioses em Juruti, intensificadas, principalmente, nos meses que antecedem as chuvas (setembro a novembro).

\section{AGRADECIMENTOS}

À Secretaria Municipal de Saúde de Juruti, pelo apoio operacional e logístico para a realização deste trabalho. À Fundação Instituto para o Desenvolvimento da Amazônia (FIDESA), pelo apoio na administração dos recursos financeiros. Ao sr. Manoel Dialma Pereira da Costa, da Secretaria Municipal de Saúde de Santarém, pelo apoio nas capturas entomológicas. Ao dr. Ricardo Guimarães, do Laboratório de Geoprocessamento do Instituto Evandro Chagas, pela produção das imagens de satélites e análises de geoprocessamento.

\section{SUPORTE FINANCEIRO}

Instituto Evandro Chagas/SVS/MS e Alcoa S/A.

\section{REFERÊNCIAS}

1 Young DG, Duncan MA. Guide to the identification and geographic distribution of Lutzomyia sand flies in Mexico, the West Indies, Central and South America (Diptera: Psychodidae). Gainesville (FL): American Entomological Institute; 1994. 881 p. (Memoirs of the American Entomological Institute; no. 54).

2 Barrett TV, Freitas RA, Albuquerque MIC, Guerrero JHC. Report on a collection of Lutzomyia sandflies (Diptera: Psychodidae) from the middle Solimões (Amazonas, Brazil). Mem Inst Oswaldo Cruz. 1996 Jan-Feb;91 (1):27-35.

3 Ward RD, Shaw JJ, Lainson R, Fraiha $H$. Leishmaniasis in Brazil: VIII. Observations on the phlebotomine fauna of an area of highly endemic cutaneous leishmaniasis in the Serra dos Carajás, Pará state. Trans R Soc Trop Med Hyg. 1973;67(2):174-83.

4 Grimaldi Jr G, Momen H, Naiff RD, McMahon-Pratt D, Barrett TV. Characterization and classification of leishmanial parasites from humans, wild mammals, and sand flies in the Amazon Region of Brazil. Am J Trop Med Hyg. 1991 Jun;44(6):645-61.

5 Rangel EF, Lainson R. Flebotomíneos do Brasil. Rio de Janeiro: FIOCRUZ; 2003. 368 p.

6 Aguiar GM, Medeiros WM. Distribuição regional e habitats das espécies de flebotomíneos do Brasil. In: Rangel EF, Lainson R, organizadores. Flebotomíneos do Brasil. Rio de Janeiro: FIOCRUZ; 2003. p. 207-55.

7 Ryan L. Flebótomos do Estado do Pará, Brasil (Diptera: Psychodidae). Belém: Instituto Evandro Chagas; 1986. 154 p. (Documento técnico; no. 1).
8 Shaw JJ, Lainson R. Trypanosoma vivax in Brazil. Ann Trop Med Parasitol. 1972;66(1):25-33

9 Fraiha $H$, Shaw JJ, Lainson R. Phlebotominae brasileiros: II - Psychodopygus wellcomei, nova espécie antropófila de flebótomo do grupo squamiventris, do Sul do Estado do Pará, Brasil (Diptera, Psychodidae). Mem Inst Oswaldo Cruz. $1971 ; 69(3): 489-500$

10 Fraiha $H$, Ward RD, Shaw JJ, Lainson R. Fauna antropofílica de flebótomos da rodovia Transamazônica, Brasil. Bol Of Sanit Panam. 1978;84(2):134-9.

11 Lainson R, Shaw JJ, Silveira FT, Braga RR. American visceral leishmaniasis: on the origin of Leishmania (Leishmania) chagasi. Trans R Soc Trop Med Hyg. 1987 May-Jun;81 (3):517.

12 Rebêlo JMM, Oliveira-Pereira YN. Flebotomíneos (Diptera, Psychodidae) de matas de terra firme e de várzea, do município de Paragominas, Estado do Pará, Brasil. Acta Amaz. 2001 mar;31(1): 145-54.

13 Souza AAA, Silveira FT, Lainson R, Barata IR, Silva MGS, Lima JAN, et al. Phlebotominae fauna in Serra dos Carajás, Pará State, Brazil, and its possible implications for the transmission of American tegumentar leishmaniasis. Rev Pan-Amaz Saude. 2010 Mar;1 (1):45-51.

14 Souza AAA, Santos TV, Jennings YLL, Ishikawa EAY, Barata IR, Silva MGS, et al. Natural Leishmania (Viannia) spp. infections in phlebotomine sand flies (Diptera: Psychodidae) from the Brazilian Amazon region reveal new putative transmission cycles of American cutaneous leishmaniasis. Parasite. 2016 May;23(22): $1-10$. 
15 Feitosa MAC, Castellon EG. Flebotomíneos (Diptera: Psychodidae) na periferia de Santarém (PA). Estratificação horizontal e fatores agravantes para transmissão domiciliar de leishmanioses. Rev Colomb Cienc Anim. 2009;1 (2):222-39.

16 Feitosa MAC, Julião GR, Costa MDP, Belém B, Pessoa FAC. Diversity of sand flies in domiciliary environment of Santarém, state of Pará, Brazil: species composition and abundance patterns in rural and urban areas. Acta Amaz. 2012;42(4):507-14.

17 Ferreira JVS, Santos TV, Santos EMG, Gorayeb IS. Phlebotomine sand flies (Diptera: Psychodidae) in forest fragments of Belém metropolitan area, Pará State, Brazil, with considerations on vectors of American cutaneous leishmaniasis agents. Rev Pan-Amaz Saude. 2014 Jun;5(2):29-35.

18 Franke CR, Staubach C, Ziller M, Schlüter H. Trends in the temporal and spatial distribution of visceral and cutaneous leishmaniasis in the state of Bahia, Brazil, from 1985 to 1999. Trans R Soc Trop Med Hyg. 2002 May-Jun;96(3):236-41.

19 Ximenes MFFM, Silva VPM, Queiroz PVS, Rego MM, Cortez AM, Batista LMM, et al. Flebotomíneos (Diptera: Psychodidae) e leishmanioses no Rio Grande do Norte, Nordeste do Brasil: reflexos do ambiente antrópico. Neotrop Entomol. 2007 jan-fev;36(1):128-37.

20 Gomes AC, Ottati SM, Shaw JJ, Lainson R, Yamamoto Yl. Active transmission of Leishmania braziliensis braziliensis in the Serra de Mar forest, São Paulo, Brazil. Trans R Soc Trop Med Hyg. 1989 Mar-Apr;83(2): 193.

21 Ministério de Minas e Energia (BR). Secretaria de Geologia, Mineração e Transformação Mineral. Produto 11: minério de alumínio, relatório técnico: perfil da mineração de bauxita [Internet]. Brasília: Ministério de Minas e Energia; 2009 [citado 2016 mai 12]. Disponível em: http://www.mme.gov.br/ documents/1 138775/1256650/P1 1 RT22 Perfil da Mineraxo de Bauxita.pdf/1713eb90-cbf9-

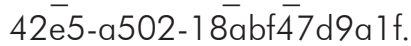

22 Soares DC, Fraiha Neto H, Garcez LM. Epidemiology of tegumentary leishmaniasis in the Municipality of Juruti, Pará State, Brazil. Rev Pan-Amaz Saude. 2010 Mar;1(1):195.

23 Garcez LM, Cardoso JF, Chagas AP, Miranda JFC, Souza GCR, Soares DC, et al. Vigilância da leishmaniose visceral em localidades epidemiologicamente distintas em Juruti, um município minerário do Estado do Pará, Brasil. Rev Pan-Amaz Saude. 2010 mar;1(1):107-16.

24 Fundação Amazônia de Amparo a Estudos e Pesquisas do Pará. Diretoria de Estatística e de tecnologia e Gestão da Informação. Estatísticas municipais paraenses: Juruti. Belém: FAPESPA; 2015. $51 \mathrm{p}$.
25 Ministério da Saúde (BR). Secretaria de Vigilância em Saúde. Departamento de Vigilância Epidemiológica. Manual de vigilância e controle da leishmaniose visceral. Brasília: Ministério da Saúde; 2014. $120 \mathrm{p}$

26 Lainson R. On Leismania enriettii and other enigmatic Leishmania species of the neotropics. Mem Inst Oswaldo Cruz. 1997 May-Jun;92(3): 377-87.

$27 \mathrm{R}$ Foundation for Statistical Computing. $\mathrm{R}$ Development Core Team, 2013. R: A language and environment for statistical computing. Vienna: $R$ Foundation for Statistical Computing; 2013.

28 Melo AS. $\bigcirc$ que ganhamos 'confundindo' riqueza de espécies e equabilidade num índice de diversidade? Biota Neot. 2008 jul-set;8(3): $21-7$.

29 Ayres M, Ayres Jr M, Ayres DL, Santos AA. BioEstat 5.0 aplicações estatísticas nas áreas das ciências biológicas e médicas. Belém: IDSM; 2007. 364 p.

30 Rebêlo JMM, Oliveira ST, Barros VLL, Silva FS. Flebotomíneos da Amazônia maranhense. III - Freqüência horária das espécies em área de colonização recente. Entom Vect. $2000 ; 7(1)$ : $1-18$.

31 Martin MCB, Rebêlo JMM. Dinâmica espaçotemporal de flebotomíneos (Diptera, Psychodidae) do município de Santa Quitéria, área de cerrado do Estado do Maranhão, Brasil. Iheringia Ser Zool. 2006 set;96(3):273-384.

32 Martins FC, Moraes JLP, Figueiredo N, Rebêlo JMM. Estrutura da comunidade de Phlebotominae (Diptera, Psychodidae) em mata ciliar do município de Urbano Santos, Maranhão, Brasil. Iheringia Ser Zool. 2011 jun;101(1-2):109-14.

33 Lainson R. Our present knowledge of the ecology and control of leishmaniasis in the Amazon Region of Brazil. Rev Soc Bras Med Trop. 1985 Jan-Mar; 18(1):47-56.

34 Ribeiro ALM, Missawa NA. Spatial distribution of phlebotomine species in the state of Mato Grosso, Brazil, in the period of 1996 to 2001 . Entom Vect. 2002;9:33-4

35 Monteiro EM, Silva JCF, Costa RT, Costa DC, Barata RA, Paula EV, et al. Leishmaniose visceral: estudo de flebotomíneos e infecção canina em Montes Claros, Minas Gerais. Rev Soc Bras Med Trop. 2005 mar-abr;38(2): 147-52.

36 Ximenes MFFM, Castellón EG, Souza MDEF, Menezes AA, Queiroz JW, Macedo VP, et al. Effect of abiotic factors on seasonal population dynamics of Lutzomyia longipalpis (Diptera: Psychodidae) in Northeastern Brazil. J Med Entomol. 2006 Sep;43(5):990-5. 
37 Oliveira AG, Galati EAB, Fernandes CE, Dorval MEC, Brazil RP. Seasonal variation of Lutzomyia longipalpis (Lutz \& Neiva, 1912) (Diptera: Psychodidae: Phlebotominae) in endemic area of visceral leishmaniasis, Campo Grande, state of Mato Grosso do Sul, Brazil. Acta Trop. 2008 Jan;105(1):55-61.

38 Oliveira EF, Silva EA, Fernandes CES, ParanhosFilho AC, Gamarra RM, Ribeiro AA, et al. Biotioc factors and occurrence of Lutzomyia longipalpis in endemic area of visceral leishmaniasis, Mato Grosso do Sul, Brazil. Mem Inst Oswaldo Cruz. 2012 May; 107(3):396-401.

39 Barata RA, França-Silva JC, Mayrink W, Silva JC, Prata A, Lorosa ES, et al. Aspectos da ecologia e do comportamento de flebotomíneos em área endêmica de leishmaniose visceral, Minas Gerais. Rev Soc Bras Med Trop. 2005 set-out;38(5):421-5.

40 Resende MC, Camargo MCV, Vieira JRM, Nobi RCA, Porto $M N$, Oliveira $C L$, et al. Seasonal variation of Lutzomyia longipalpis in Belo Horizonte, State of Minas Gerais. Rev Soc Bras Med Trop. 2006 Jan-Feb;39(1):51-5.

41 Alexander B, Carvalho RL, Mccallum H, Pereira $\mathrm{MH}$. Role of the domestic chicken (Gallus gallus) in the epidemiology of urban visceral leishmaniasis in Brazil. Emerg Infect Dis. 2002 Dec;8(12):1480-5.

42 Santini MS, Salomon OD, Acardi SA, Sandoval EA, Tartaglino L. Lutzomyia longipalpis behavior and control at an urban visceral leishmaniasis focus in Argentina. Rev Inst Med Trop S Paulo. 2010 Jul-Aug;52(4):187-91.

43 Martins AV, Williams P, Falcão AL. American sand flies (Diptera: Psychodidae: Phlebotominae). Rio de Janeiro: Academia Brasileira Ciências; 1978. $195 \mathrm{p}$.

44 Feliciangeli MD. Taxonomic review of Lutzomyia walkeri (Newstead, 1914) (= Lutzomyia marajoensis (Damasceno \& Causey, 1944) and the resurrection of Lutzomyia dubitans (Sherlock, 1962) (Diptera: Psychodidae). Mem Inst Oswaldo Cruz. 1985 Jul-Sep;80(3):307-12.
45 Silva DF, Freitas RA, Franco AMR. Diversidade e abundância de flebotomíneos do gênero Lutzomyia (Diptera: Psychodidae) em áreas de mata do nordeste de Manacapuru, AM. Neotrop Entomol. 2007 jan-fev;36(1):138-44.

46 Silva DF, Vasconcelos SD. Flebotomíneo em fragmentos de Mata Atlântica na Região Metropolitana do Recife, PE. Rev Soc Bras Med Trop. 2005 mai-jun;38(3):264-6.

47 Rebêlo JMM, Oliveira ST, Silva FS, Barros VLL, Costa JML. Sandflies (Diptera: Psychodidae) of the Amazônia of Maranhão. V. Seasonal occurrence in ancient colonization area and endemic for cutaneous leishmaniasis. Rev Bras Biol. 2001 Feb;61 (1):107-15.

48 Rebêlo JMM, Oliveira ST, Barros VLL, Silva FS. Flutuação sazonal dos flebotomíneos (Diptera, Psychodidae) em área de colonização recente do município de Buriticupu, Amazônia maranhense, Brasil. Rev Bras Entomol. 2001;45(1):11-6.

49 Rebêlo JMM. Freqüência horária e sazonalidade de Lutzomyia longipalpis (Diptera: Psychodidae: Phlebotominae) na llha de São Luís, Maranhão, Brasil. Cad Saude Publica. 2001 jan-fev;17(1): $221-7$.

50 Barata RA, Silva JCF, Costa RT, Fortes-Dias CL, Silva JC, Paula EV, et al. Phlebotomine sand flies in Porteirinha, an area of American visceral leishmaniasis transmission in the State of Minas Gerais, Brazil. Mem Inst Oswaldo Cruz. 2004 Aug;99(5):481-7.

51 Rutledge LC, Ellenwood DA. Production of phlebotomine sandflies on the open forest floor in Panama: the species complement. Environ Entomol. 1975 Feb;4(1):71-7. 